\title{
Combining Step Gradients and Linear Gradients in Density
}

\section{Citation}

Kumar, Ashok A., Jenna A. Walz, Mathieu Gonidec, Charles R. Mace, and George M. Whitesides. 2015. "Combining Step Gradients and Linear Gradients in Density." Anal. Chem. 87 (12) (June 16): 6158-6164. doi:10.1021/acs.analchem.5b00763.

\section{Published Version}

doi:10.1021/acs.analchem.5b00763

\section{Permanent link}

http://nrs.harvard.edu/urn-3:HUL.InstRepos:25042502

\section{Terms of Use}

This article was downloaded from Harvard University's DASH repository, and is made available under the terms and conditions applicable to Open Access Policy Articles, as set forth at http:// nrs.harvard.edu/urn-3:HUL.InstRepos:dash.current.terms-of-use\#OAP

\section{Share Your Story}

The Harvard community has made this article openly available.

Please share how this access benefits you. Submit a story.

Accessibility 


\title{
Combining Step-Gradients and Linear Gradients in Density
}

\author{
Ashok A. Kumar ${ }^{1}$, Jenna A. Walz ${ }^{2}$, Mathieu Gonidec ${ }^{1}$, Charles R. Mace ${ }^{2}$, and \\ George M. Whitesides ${ }^{1,3,4^{*}}$
}

${ }^{1}$ Department of Chemistry and Chemical Biology, Harvard University, Cambridge, MA 02138, ${ }^{2}$ Department of Chemistry, Tufts University, Medford, MA 02115, ${ }^{3}$ Wyss Institute for Biologically Inspired Engineering, ${ }^{4}$ Kavli Institute for Bionano Science and Technology, Harvard University, Cambridge, MA 02138

*Corresponding author: gwhitesides@gmwgroup.harvard.edu 


\section{Abstract}

Combining aqueous multiphase systems (AMPS) and magnetic levitation (MagLev) provides a method to produce hybrid gradients in apparent density. AMPS — solutions of different polymers, salts, or surfactants that spontaneously separate into immiscible but predominantly aqueous phases_-offer thermodynamically stable steps in density that can be tuned by the concentration of solutes. MagLev - the levitation of diamagnetic objects in a paramagnetic fluid within a magnetic field gradient — can be arranged to provide a near-linear gradient in effective density where the height of a levitating object above the surface of the magnet corresponds to its density; the strength of the gradient in effective density can be tuned by the choice of paramagnetic salt and its concentrations, and by the strength and gradient in the magnetic field. Including paramagnetic salts (e.g., $\mathrm{MnSO}_{4}$ or $\mathrm{MnCl}_{2}$ ) in AMPS, and placing them in a magnetic field gradient, enables their use as media for MagLev. The potential to create large steps in density with AMPS allows separations of objects across a range of densities. The gradients produced by MagLev provide resolution over a continuous range of densities. By combining these approaches, mixtures of objects with large differences in density can be separated and analyzed simultaneously. Using MagLev to add an effective gradient in density also enables tuning the range of densities captured at an interface of an AMPS by simply changing the position of the container in the magnetic field. Further, by creating AMPS in which phases have different concentrations of paramagnetic ions, the phases can provide different resolutions in density. These results suggest that combining steps in density with gradients in density can enable new classes of separations based on density.

\section{Introduction}

The separation of a heterogeneous mixture into sub-populations is a common task in science. Methods for separation often use a property that is common to all the components of 
each sub-population. Techniques based on size (e.g., size-exclusion chromatography $)^{1,2}$ and on affinity (for biomolecules) $)^{3-6}$ are used routinely in separations. Density, a physical property of all matter, is also a property that is useful in separations. ${ }^{5,7}$ Table 1 provides examples of applications of density-based separations and analyses.

Applications that require sorting (e.g., archaeology or recycling) benefit from methods to separate objects by density in order to segregate mixtures into bins with distinct steps in density. The simplest method is "sink-float": objects denser than the medium sink and separate from those that are less dense, which float. Many applications in separations, however, require gradients in density to resolve subtle differences within and among populations in a sample (e.g., analysis of forensic evidence) ${ }^{8}$ There are two primary types of gradients in density that have been used experimentally: continuous gradients of densitywhere each position in, for example, a column of medium represents a different density — and steps in density—where a range of densities are binned between two solutions of different densities. ${ }^{9}$ Methods to create continuous gradients in density include centrifugation of a polydisperse colloidal suspension (i.e., Percoll) ${ }^{9}$ and the introduction of a paramagnetic fluid into a magnetic field gradient (i.e., magnetic levitation). ${ }^{10}$ Methods to create discontinuous steps in density include layered media (e.g., sucrose gradients) ${ }^{11}$ and phase separation of immiscible solutions (e.g., aqueous multiphase systems [AMPS]). ${ }^{12}$ Gradients in density are typically used with centrifugation, ${ }^{9,13}$ and sometimes ultracentrifugation. ${ }^{14}$

Combining the sensitivity of continuous gradients with the dynamic range of steps in density can enable the simultaneous separation of two or more populations whose mean densities differ by large amounts, but whose subpopulations have small differences in density. Further, this approach can enable the separation and analysis of objects over different ranges of density with different sensitivities. This paper describes the combination of linear gradients in density, with step-gradients in density, by a technique that uses magnetic 
Table 1. Examples of Density-based Separations and Analyses.

\begin{tabular}{|c|c|c|c|c|}
\hline Target & Background & Application & Densities $\left(\mathrm{g} / \mathrm{cm}^{3}\right)$ & Ref. \\
\hline bacteria & bacterial colonies, cultures & $\begin{array}{l}\text { medical diagnostics, } \\
\text { biological research }\end{array}$ & $1.09-1.11$ & 15,16 \\
\hline biodiesel and fuels & fuel blends & $\begin{array}{l}\text { monitoring blends and } \\
\text { quality of fuels }\end{array}$ & $0.73-1.02$ & 17,18 \\
\hline blood cells & blood & $\begin{array}{l}\text { medical diagnostics, } \\
\text { biological research }\end{array}$ & $1.06-1.22$ & $19-21$ \\
\hline carbon nanotubes & mixtures of carbon nanotubes & electronics, optics, sensing & $1.05-1.30$ & $14,22,23$ \\
\hline food products & $\begin{array}{l}\text { oil, milk, cheese, peanut } \\
\text { butter, grains }\end{array}$ & monitoring fat content & $0.91-1.00$ & 24,25 \\
\hline glitter & samples from crime scenes & forensic evidence & $1.20-2.50$ & 8,26 \\
\hline healthy sperm & semen & fertilization & $1.10-1.25$ & 27,28 \\
\hline organelles & cell lysate & metabolic research & $1.13-1.26$ & 29,30 \\
\hline defective parts & large-scale manufactured parts & quality control & $0.91-2.99$ & 31 \\
\hline solid polymers & $\begin{array}{l}\text { mixtures of different } \\
\text { chemically modified solid } \\
\text { polymers }\end{array}$ & $\begin{array}{l}\text { purification, quality } \\
\text { control, monitoring } \\
\text { reactions }\end{array}$ & $0.91-1.42$ & 32 \\
\hline $\begin{array}{l}\text { bones with specific } \\
\text { mineral content }\end{array}$ & mixtures of bones & archaeology, anthropology & $1.80-2.40$ & 33 \\
\hline waste streams & plastics, plastic foams & recycling & $0.03-2.20$ & 7,34 \\
\hline
\end{tabular}


levitation in AMPS containing a paramagnetic solute (e.g., $\mathrm{Mn}(\mathrm{II}) \mathrm{Cl}_{2}$ ). This combination allows the step in density of an AMPS to be tuned by the position of a liquid/liquid interface in a magnetic field. We apply this capability to capture different plastics (polystyrene and Nylon) at an interface by simply varying the height of a container between two magnets.

\section{Magnetic levitation provides a linear gradient in density.}

When suspended in a solution containing paramagnetic ions, and placed in a magnetic field gradient, diamagnetic materials (e.g., glass or plastics) can levitate (Figure 1). At equilibrium, the effective magnetic force $\left(F_{m}\right)$ acting on the diamagnetic material balances the gravitational force $\left(F_{g}\right)$ in the vertical direction at a height, $h(\mathrm{~m})$, which we call the "levitation height." When the orientation and strengths of the permanent NdFeB magnets used to generate the field gradient provide a linear gradient along the vertical axis, the balance of forces creates a linear gradient in effective density. That is, the gradient is a result of the balance of magnetic and gravitational forces rather than purely a balance of gravitational forces. A diamagnetic object—which we assume to experience a negligible magnetic force - experiences a gradient that is effectively the same as being placed in a linear gradient formed by solutions of density that varies with position. ${ }^{10} \mathrm{We}$ express the levitation height approximately as a function of the physical properties of the diamagnetic material, the paramagnetic solution, and the MagLev device using Equation 1 (we give complete treatments of this subject elsewhere $\left.{ }^{10}\right)$ :

$$
h=\frac{\left(\rho_{s}-\rho_{m}\right) g \mu_{0} d^{2}}{\left(\chi_{s}-\chi_{m}\right) 4 B_{0}^{2}}+\frac{d}{2}
$$

In Equation 1, $\rho_{m}\left(\mathrm{~kg} / \mathrm{m}^{3}\right)$ is the density of the paramagnetic medium (we typically represent density in the units of $\mathrm{g} / \mathrm{cm}^{3}$ for convenience; $\left.10^{-3} \mathrm{~g} / \mathrm{cm}^{3}=1 \mathrm{~kg} / \mathrm{m}^{3}\right), \rho_{s}\left(\mathrm{~kg} / \mathrm{m}^{3}\right)$ is the density of the levitating material, $g$ is the acceleration due to gravity $\left(9.81 \mathrm{~m} / \mathrm{s}^{2}\right), \chi_{m}$ and $\chi_{s}$ (both unitless) are the magnetic susceptibilities of the paramagnetic medium and the 
Figure 1. Schematic representations of the behavior of objects in magnetic levitation and in aqueous multiphase systems (AMPS). (A) Three diamagnetic objects levitate at different heights depending on the position at which the magnetic and gravitational forces $\left(\mathrm{F}_{\mathrm{m}}\right.$ and $\left.\mathrm{F}_{\mathrm{g}}\right)$ acting on the objects are in equilibrium. (B) In an AMPS, objects either float, sink, or come to rest at a liquid-liquid interface. (C) In a paramagnetic AMPS in a magnetic field gradient, a combination of behaviors is possible. Objects 1 and 3 levitate in the phases while object 2 is trapped at the liquid-liquid interface. In this example, the interface between phases is located at the midpoint $(\mathrm{d} / 2)$ of the distance between the magnets.

A

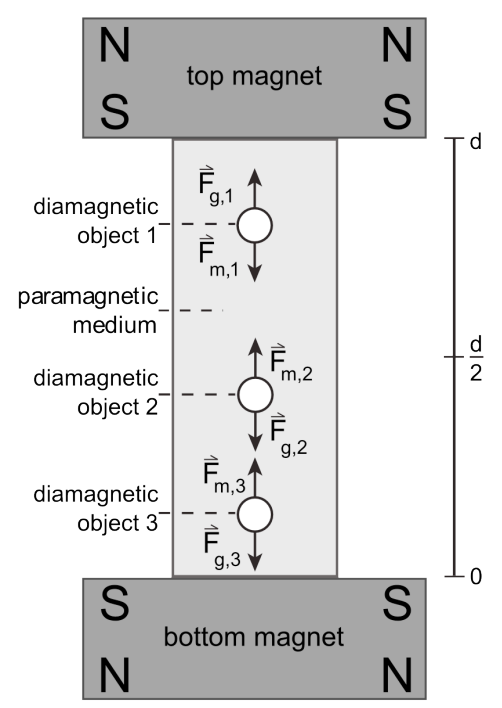

B

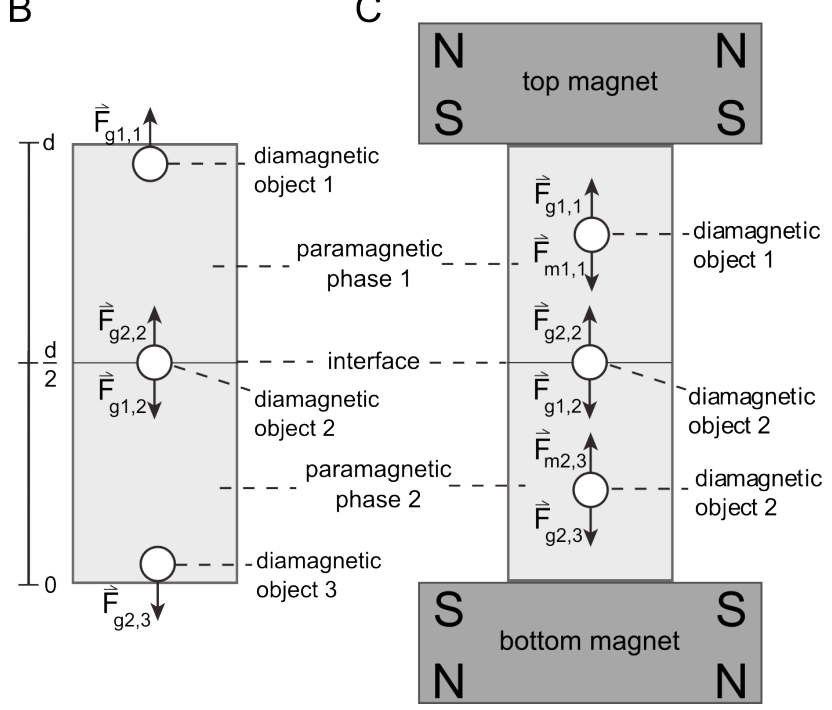

C 
suspended material, respectively, $\mu_{0}\left(1.26 \times 10^{-6}[\mathrm{~kg} \cdot \mathrm{m}] /\left[\mathrm{A}^{2} \cdot \mathrm{s}^{2}\right]\right)$ is the magnetic permeability of free space, and $B_{0}\left(\mathrm{~T}=\mathrm{kg} /\left[\mathrm{A} \cdot \mathrm{s}^{2}\right]\right)$ is the magnetic field strength at the surface of the magnet. Equation 1 is derived for a point-particle, but the equation is a good approximation for objects of finite volume where $h$ is the height of the centroid of the object. ${ }^{35}$

Aqueous multiphase systems provide steps in density.

AMPS are prepared from solutions of polymers, salts, or surfactants using mixtures that spontaneously separate into immiscible equilibrium phases. ${ }^{12}$ Two-phase AMPS are a subclass of AMPS that are often called aqueous two-phase systems (ATPS). ${ }^{36,37}$ The diversity of polymers, surfactants, and salts that are mutually immiscible allows the creation of hundreds of multiphase systems. ${ }^{12}$

Phases in AMPS order in a gravitational or centrifugal field according to their densities, and the sharp interface between phases provides a stable, essentially molecularly sharp, step in density. Objects will rest at the interface between two phases if the density of the object is between the densities of the two phases (i.e., $\rho_{\text {upper }}<\rho_{\text {object }}<\rho_{\text {lower }}$ ). ${ }^{12}$ The density of each phase can be tuned by the choice and concentration of polymers, and increased by the addition of co-solutes, such as salts. The useful size of the steps in density between phases can range from $<0.001 \mathrm{~g} / \mathrm{cm}^{3}$ to $>0.100 \mathrm{~g} / \mathrm{cm}^{3}$. The step-gradient in density formed by AMPS are also capable of separating mammalian cells by density. ${ }^{20,21}$ The interface between phases can represent a step in other physical properties, such as viscosity ${ }^{38}$, index of refraction, or — when paramagnetic salts are included as co-solutes - magnetic susceptibility.

We have combined AMPS and MagLev to create separation media whose steps and gradients in density — in a magnetic field — are stable, time-invariant, and tunable in both absolute density and resolution in density. Here, we demonstrate phases with over a five-fold difference in resolution in density. 


\section{Experimental Design}

\section{Selection of Levitation Conditions}

We describe the MagLev device elsewhere in detail. ${ }^{10}$ Briefly, two NdFeB permanent magnets $(5.1 \mathrm{~cm} \times 5.1 \mathrm{~cm} \times 2.5 \mathrm{~cm})$ are positioned with like poles facing. The field strength at the surface of the magnets is $0.371 \mathrm{~T}$. We use a distance between the magnets of $46 \mathrm{~mm}$ to accommodate a standard spectrophotometer cuvette (as the container for the paramagnetic medium). This geometry with these magnets also generates the near-linear gradient in the magnetic field necessary for Equation 1 to be valid. ${ }^{10}$

\section{Selection of Aqueous Multiphase Systems}

The large selection of polymers, salts, and surfactants known to produce AMPS allowed us to choose a system that provided a desirable combination of step and linear gradients in density. ${ }^{12,39,40}$ Salts (or other solutes) may partition between the different phases of AMPS depending on their relative affinity for each phase. We can use this behavior to design gradients in density. For example, if a paramagnetic salt partitions preferentially into the top phase of a two-phase AMPS, then the effective gradient in density will have a greater slope in the top phase than the bottom phase (when placed in a linear gradient in a magnetic field). To demonstrate this capability, we sought two different AMPS: one in which partitioning of the paramagnetic ions between phases would be negligible and one in which the partitioning would be large.

Based on previous work demonstrating that chloride salts in AMPS with neutral polymers have little effect on phase separation ${ }^{41}$ and partition evenly between phases, ${ }^{42}$ we expected that such systems would be a good candidate for a system where a paramagnetic chloride salt would partition approximately equally between both phases; in such a system, water would be the predominant component in both phases, and we expected the interactions 
of the salt with the neutral polymers would be minimally different. We, thus, chose to use poly(ethylene glycol) (PEG) and dextran to create a system with similar gradients in each phase.

An extreme case of salt partitioning between phases occurs when a polymer and a salt phase separate. Solutions of high concentrations of metal sulfates mixed with PEG phase separate and form AMPS with a much higher concentration of salt in one phase than the

other. ${ }^{43-45}$ We predicted that a large step in magnetic susceptibility could be formed in an AMPS comprising a polymer, such as PEG, and a paramagnetic sulfate salt, such as $\mathrm{MnSO}_{4}$. We, thus, chose a PEG- $\mathrm{MnSO}_{4}$ system to explore a gradient with a large step in the magnetic susceptibility.

\section{Analysis of Aqueous Multiphase Systems}

We prepared the PEG-dextran AMPS using polymers with different molecular weights to facilitate the characterization of each phase by gel permeation chromatography (Supporting Information). The molecular weights used in the PEG-dextran system were $20 \mathrm{kDa}$ and $500 \mathrm{kDa}$, respectively. The molecular weight of the PEG used in the PEG$\mathrm{MnSO}_{4}$ system was $1.5 \mathrm{kDa}$. We used a U-tube densitometer (DMA 35, Anton Paar) to measure the density of each phase of the AMPS. Flame atomic absorption spectroscopy (FAA) quantified the concentration of manganese in each phase of the AMPS. We measured the intensity of the Mn-specific wavelength $(\lambda=257.610 \mathrm{~nm})$ from diluted aliquots of each phase. We compared these intensities to those from a standard curve that we generated using known concentrations of $\mathrm{MnCl}_{2}$ or $\mathrm{MnSO}_{4}$.

If the polymers coordinate with the manganese ions, slight changes in the coordination geometry can occur; these changes might affect the effective magnetic susceptibility of the ions by changing their g-factor. ${ }^{46}$ Measuring the magnetic susceptibility 
by the Evans method with NMR allowed us to assess our systems for effects from interactions between the ions and the polymers. ${ }^{47,48}$

Using the magnetic susceptibility measured by Evans method, the concentration of $\mathrm{Mn}^{2+}$ measured by FAA, the density of the phases, and the positions of an object suspended by MagLev, we can measure the density of the object using Equation 1 . The density of the object can be estimated without using the Evans method if one assumes the magnetic susceptibility of the solutions will be the same as that of $\mathrm{MnCl}_{2}$ in a purely aqueous solution. In the case of the systems we present here, the additional correction provided by the Evans method provides only a slight improvement $(\sim 0.1 \%)$ in the accuracy of the measurement of density using density-standard glass beads (Table S-1). Table S-2 lists the final compositions and characterization of the two AMPS studied in this work.

\section{Estimations of the Density of Beads using Magnetic Levitation}

We used the characteristics of the phases, images of beads levitating in a magnetic field, and Equation 1 to calculate the densities of beads (see SI for details). We used a DC magnetometer (AlphaLab) to measure the surface field of the magnets. Measuring the height of levitation requires finding the centroid of each bead. Due to the slightly oblong and nonspherical shape of some of the beads, we found the centroid by tracing the outline of each bead and using digital analysis (ImageJ) to calculate a centroid from the outline. We estimated the uncertainty in each density we calculated by assuming each variable in Equation 1 was independent and estimating the uncertainty in each measurement either by taking the standard deviation of multiple $(n \geq 7)$ measurements or using the accuracy of the measurement method (see SI for details). 


\section{Results and Discussion}

\section{AMPS and MagLev Provide Hybrid Gradients that are Thermodynamically Stable}

Each phase of the AMPS occupies a range of available levitation heights. The total range of buoyant density available to each phase, if used as a separate levitation medium, is truncated at the interface due to the step in density between phases. This truncation results in a gap of buoyant density that is related to the difference in density between phases, and to their difference in magnetic susceptibility (Figure 2). The magnitude of the step in density can be tuned by changing the concentrations of the polymers used to produce the AMPS, or by selecting a different set of immiscible solutes. ${ }^{12}$

We prepared an AMPS from a mixture with a final concentration of 5\% (wt/vol) PEG $(\mathrm{MW}=20 \mathrm{kDa}), 10 \%(\mathrm{wt} / \mathrm{vol})$ dextran $(\mathrm{MW}=500 \mathrm{kDa})$, and $315 \mathrm{mM} \mathrm{MnCl}_{2}$. We provide details on how to prepare the AMPS in this work in the Supporting Information. The top phase of the resulting AMPS was enriched significantly for PEG and contained $323 \mathrm{mM}$ $\mathrm{Mn}^{2+}$; the bottom phase was enriched for dextran and contained $305 \mathrm{mM} \mathrm{Mn}^{2+}$ (Supporting Information). The effective gradient in buoyant density generated by placing the AMPS in a MagLev device, therefore, was approximately constant throughout the medium $(\nabla \rho=$ $0.00100 \mathrm{~g} / \mathrm{cm}^{3} / \mathrm{mm}$ and $0.00105 \mathrm{~g} / \mathrm{cm}^{3} / \mathrm{mm}$ for the top and bottom phases, respectively). Despite having similar slopes in density, the density of each phase is different and, thus, each phase provides access to a different range of effective densities (Table S-3).

\section{Separating Beads Based on Density with MagLev and AMPS}

To the PEG-dextran AMPS, we added a set of five reference beads with standardized densities claimed (by the supplier) to be $1.020 \mathrm{~g} / \mathrm{cm}^{3}$ (green), $1.040 \mathrm{~g} / \mathrm{cm}^{3}$ (red), $1.076 \mathrm{~g} / \mathrm{cm}^{3}$ (yellow), $1.120 \mathrm{~g} / \mathrm{cm}^{3}$ (amber), and $1.250 \mathrm{~g} / \mathrm{cm}^{3}$ (green). In the absence of an external magnetic field, the beads are confined to three positions in the AMPS (Figure 3A). Within the MagLev device, the beads separate at a height that is proportional to their densities 
Figure 2. Schematic representation of possible gradients attainable with MagLev and aqueous multiphase systems (AMPS). MagLev creates a linear gradient in effective density (A). AMPS without paramagnetic salts provide a sharp step gradient in density (B). The interface (i) can be placed at various heights $(B, C$, and D) and can create a small $(\Delta \rho \approx 0.025$ $\left.\mathrm{g} / \mathrm{cm}^{3}, \mathrm{C}\right)$ or large $\left(\Delta \rho \approx 0.080 \mathrm{~g} / \mathrm{cm}^{3}, \mathrm{D}\right)$ step in density between the top (t) and bottom (b) phases. Paramagnetic ions dissolved in the phases of AMPS create near-linear gradients in effective density separated by a step in density $(\mathrm{C}$ and $\mathrm{D})$. Partitioning of paramagnetic ions between phases of an AMPS can create equal (C) or unequal (D) gradients in effective density. In both (C) and (D) there is a range of densities (grey boxes) where density cannot be measured as a function of height.

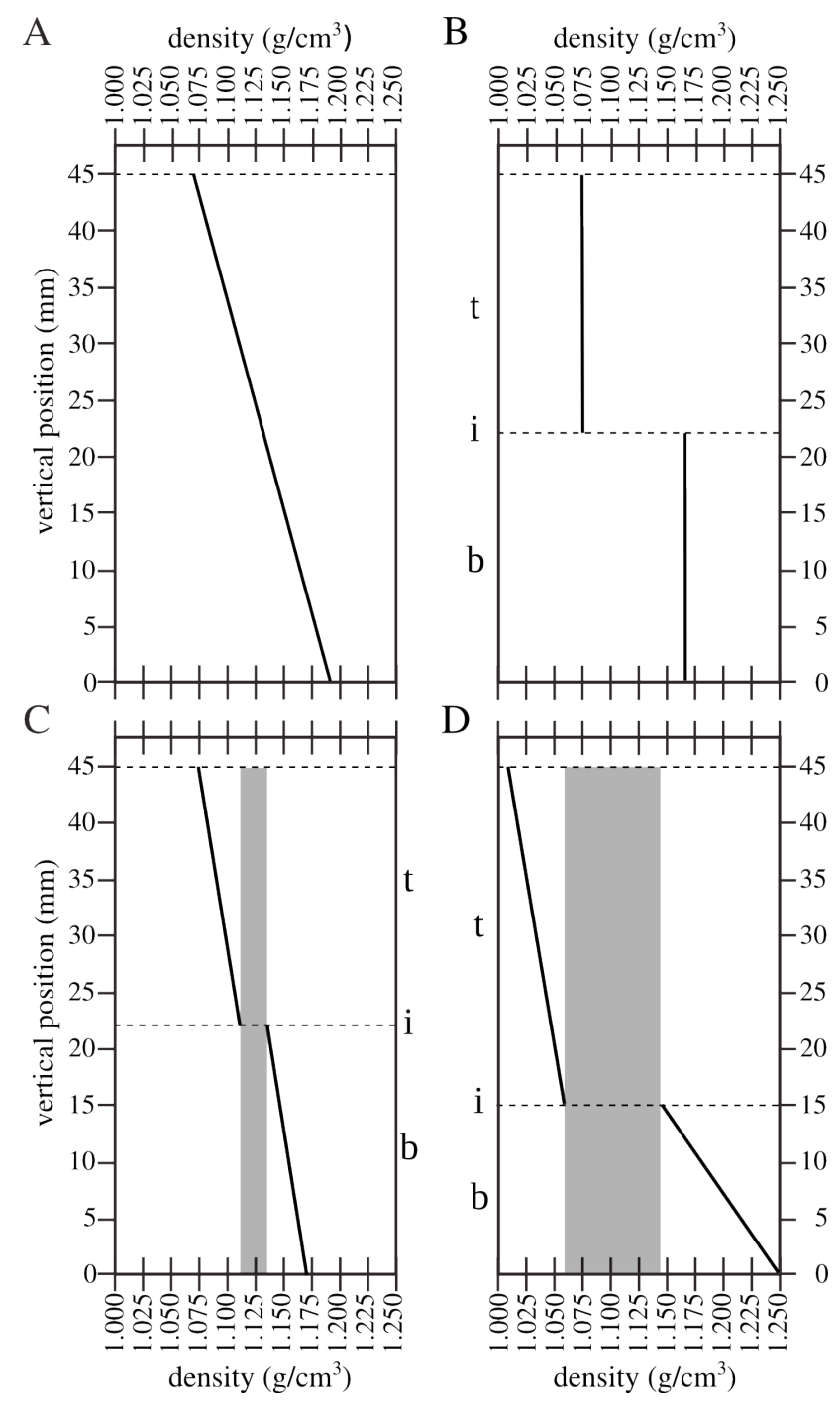


Figure 3. Preparing stable steps and gradients in density using aqueous multiphase systems (AMPS) as media for magnetic levitation (MagLev). A mixture of 5\% (wt/vol) poly(ethylene glycol), 10\% (wt/vol) dextran, and $315 \mathrm{mM} \mathrm{MnCl}_{2}$ demonstrates the difference between (A) a step gradient and (B) a combination of a step and a linear gradient. The gradients in density are illustrated by five density standard beads: $1.020 \mathrm{~g} / \mathrm{cm}^{3}$ (green, top), $1.040 \mathrm{~g} / \mathrm{cm}^{3}$ (red), $1.076 \mathrm{~g} / \mathrm{cm}^{3}$ (yellow), $1.120 \mathrm{~g} / \mathrm{cm}^{3}$ (amber), and $1.250 \mathrm{~g} / \mathrm{cm}^{3}$ (green, bottom). MagLev can be used to measure the densities of beads that levitate within a phase of the AMPS. Heights for levitating and floating beads are estimated by calculating the centroid of each bead. Beads at a fluid interface (black arrows, liquid/air and liquid/liquid) rest at a height based on the combination of surface forces, magnetic forces, and gravitational forces. Beads at the solid/liquid interface (white arrows) rest at the surface of the inside of the container. 
A. density $\left(\mathrm{g} / \mathrm{cm}^{3}\right)$

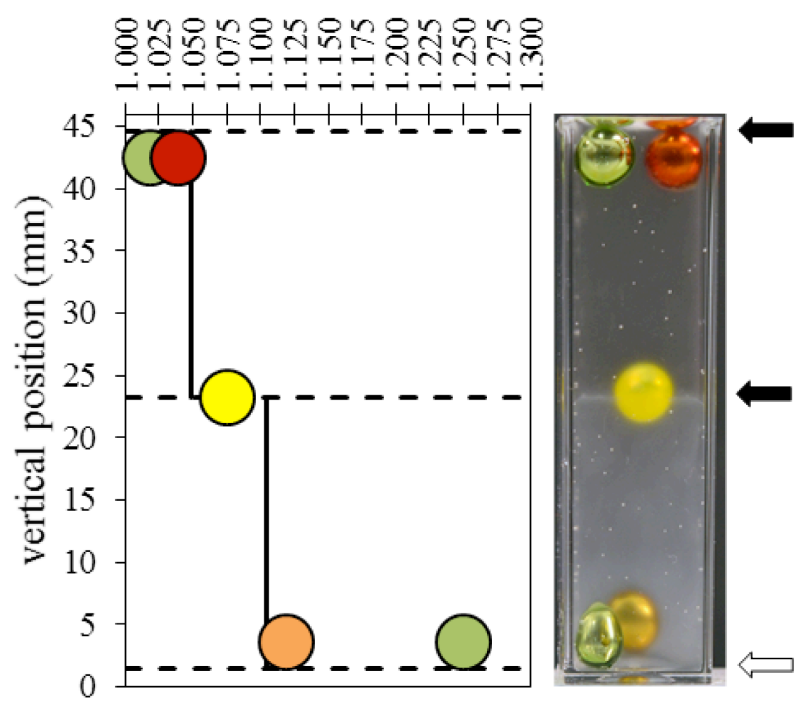

B.

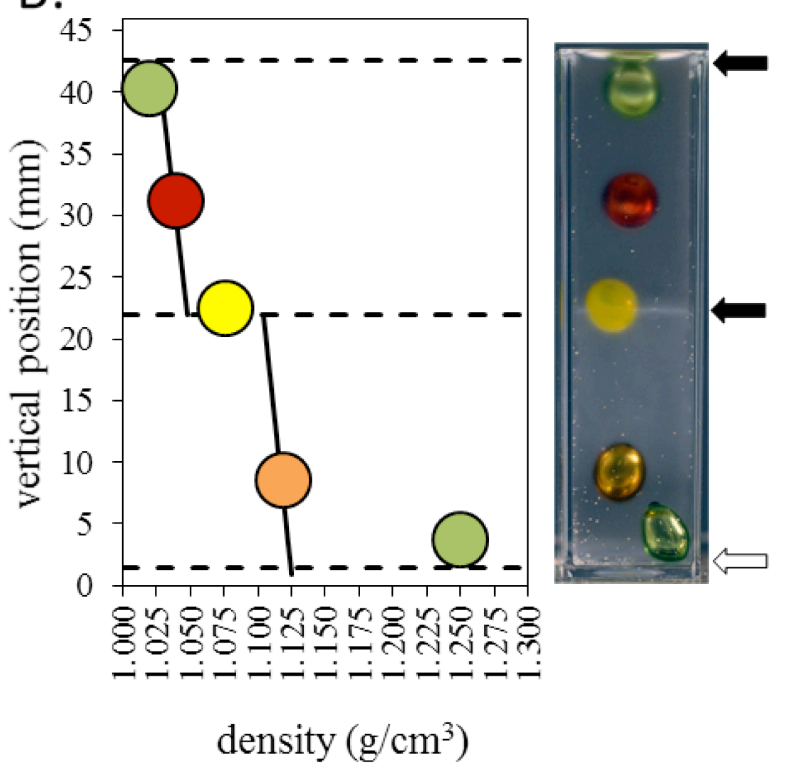


(Figure 3B). The position of three beads - with densities of $1.020 \mathrm{~g} / \mathrm{cm}^{3}, 1.076 \mathrm{~g} / \mathrm{cm}^{3}$, and $1.250 \mathrm{~g} / \mathrm{cm}^{3}$ - did not change upon introduction of the magnetic field gradient. The remaining two beads, however, reached a stable levitation height within a phase of the AMPS. Using the measured levitation heights, we calculated the densities of these beads to be $1.040 \pm 0.001 \mathrm{~g} / \mathrm{cm}^{3}$ and $1.120 \pm 0.001 \mathrm{~g} / \mathrm{cm}^{3}$; these measurements are in excellent agreement with their known densities of $1.040 \mathrm{~g} / \mathrm{cm}^{3}$ and $1.120 \mathrm{~g} / \mathrm{cm}^{3}$. This agreement demonstrates the use of the phases of an AMPS as media for MagLev separations and analyses.

\section{Forming Unequal Gradients in Density}

The effect of unequal partitioning of $\mathrm{Mn}^{2+}$ between phases was amplified further in an AMPS formed with a polymer and a salt. We prepared a mixture with a final concentration of $16.7 \%(\mathrm{wt} / \mathrm{vol})$ PEG $(\mathrm{MW}=1.5 \mathrm{kDa})$ and $875 \mathrm{mM} \mathrm{MnSO}_{4}$ (details in the Supporting Information). The top phase of the AMPS was significantly enriched for PEG and contained $327 \mathrm{mM} \mathrm{Mn}^{2+}$; the bottom phase was predominantly salt with a final concentration of 1,496 $\mathrm{mM} \mathrm{Mn}^{2+}$. In this AMPS, the gradients in buoyant density were $0.00104 \mathrm{~g} / \mathrm{cm}^{3} / \mathrm{mm}$ and $0.00584 \mathrm{~g} / \mathrm{cm}^{3} / \mathrm{mm}$ for the PEG-rich and $\mathrm{MnSO}_{4}$-rich phases, respectively.

To demonstrate MagLev separations and analyses using the large difference in magnetic susceptibility resulting from the difference in the concentration of $\mathrm{Mn}^{2+}$ between phases, we added a series of five reference beads with densities of $1.045 \mathrm{~g} / \mathrm{cm}^{3}$ (yellow), $1.081 \mathrm{~g} / \mathrm{cm}^{3}$ (red), $1.100 \mathrm{~g} / \mathrm{cm}^{3}$ (clear), $1.250 \mathrm{~g} / \mathrm{cm}^{3}$ (green), and $1.500 \mathrm{~g} / \mathrm{cm}^{3}$ (clear) to the PEG-MnSO 4 AMPS (Figure 4). When we placed this system in the magnetic field, two beads reached a stable levitation height within a phase of the AMPS and could be analyzed by MagLev. We calculated the densities of these beads to be $1.079 \pm 0.002 \mathrm{~g} / \mathrm{cm}^{3}$ and $1.249 \pm$ $0.002 \mathrm{~g} / \mathrm{cm}^{3}$. The calculated densities of these beads are in very good agreement with their claimed densities of $1.081 \mathrm{~g} / \mathrm{cm}^{3}$ and $1.250 \mathrm{~g} / \mathrm{cm}^{3}$. Based on the physical properties of this 
Figure 4. Preparing unequal gradients in density using an aqueous multiphase system (AMPS) formed from a paramagnetic salt as a medium for magnetic levitation (MagLev). A mixture of $16.7 \%$ (wt/vol) poly(ethylene glycol) and $875 \mathrm{mM} \mathrm{MnSO}_{4}$ generates an AMPS with a large step in both density and magnetic susceptibility between phases. The gradients in density are illustrated by five density standard beads: $1.045 \mathrm{~g} / \mathrm{cm}^{3}$ (yellow), $1.081 \mathrm{~g} / \mathrm{cm}^{3}$ (red), $1.100 \mathrm{~g} / \mathrm{cm}^{3}$ (clear, middle), $1.250 \mathrm{~g} / \mathrm{cm}^{3}$ (green), and $1.500 \mathrm{~g} / \mathrm{cm}^{3}$ (clear, bottom). MagLev can be used to measure the densities of beads that levitate within a phase of the AMPS (red and green bead). We measure the height of each bead by calculating the centroid for each bead. Beads that contact a fluid interface (black arrows, air/liquid and liquid/liquid) rest at a height based on the combination of surface forces, magnetic forces, and gravitational forces. Beads at the solid/liquid interface (white arrow) at the bottom of the container rest on inside surface of the container.

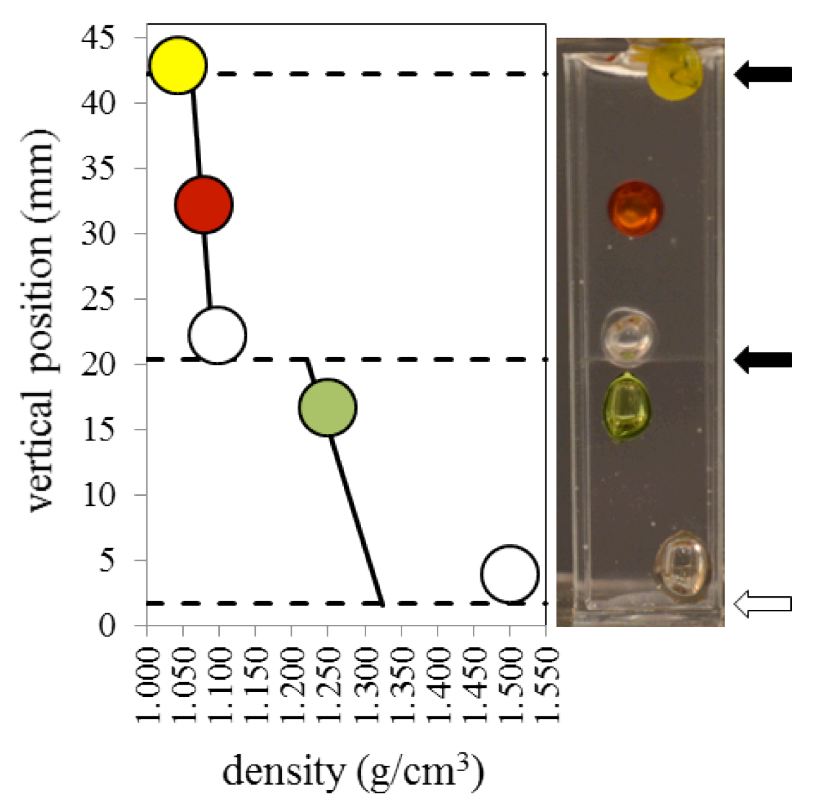


PEG-MnSO 4 AMPS (Table S-4), measurements of the densities of both beads could not be performed simultaneously in either of the individual phases. The use of a two-phase system enables the separation and concurrent analysis of objects with such a large difference in density. In this case, we were able to effectively probe a broader range of density of $1.063-$ $1.350 \mathrm{~g} / \mathrm{cm}^{3}$ with the two phase system than we could access with each phase alone (1.063$1.118 \mathrm{~g} / \mathrm{cm}^{3}$ or $\left.1.077-1.350 \mathrm{~g} / \mathrm{cm}^{3}\right)$ (Table $\left.\mathbf{S}-4\right)$.

\section{Tuning the Step in Density of an AMPS with MagLev}

In an AMPS without paramagnetic phases, the step in density at the interface between phases is defined by the concentrations of solutes in each phase and can only be adjusted by changing the composition of the system. In a paramagnetic AMPS, the effective step in density at an interface is also dependent on the paramagnetic susceptibility of each phase and the position of the interface in a magnetic field gradient (Figure 5). The ability to tune the step in density in an AMPS by simply changing the position of the container holding the paramagnetic solution and diamagnetic sample allows a system to trap different populations at an interface without changing solutions.

To demonstrate an application of this ability to tune the step in density, we designed a paramagnetic AMPS to separate two different polymers (polystyrene and Nylon) (Figure 5) (details in the Supporting Information). We used an AMPS of 4.5\% (wt/vol) PEG (MW = 20 $\mathrm{kDa})$ and $9.0 \%(\mathrm{wt} / \mathrm{vol})$ dextran $(\mathrm{MW}=500 \mathrm{kDa})$ with $584 \mathrm{mM}$ of $\mathrm{MnCl}_{2}$. When the cuvette is placed near the top of the MagLev device, the liquid/liquid interface trapped low-density $\left(\rho_{\mathrm{p} 1}=1.050, \rho_{\mathrm{p} 2}=1.051\right)$ polystyrene beads at the interface (Figure 5). When the cuvette is lowered to the bottom of the MagLev device, the liquid/liquid interface shifted to a capture the higher-density $\left(\rho_{\mathrm{n} 1}=1.126 \mathrm{~g} / \mathrm{cm}^{3}, \rho_{\mathrm{n} 2}=1.135 \mathrm{~g} / \mathrm{cm}^{3}\right)$ Nylon beads (Figure 5). In MagLev alone, we would not be able to bin the two Nylon beads at the same location because of their difference in density. With AMPS alone, we could not use the same system to capture 
Figure 5. The step in density at the interface of a paramagnetic AMPS is dependent on the height of the interface in a MagLev device. When a small cuvette is at the bottom of the MagLev device, blue Nylon beads (blue " $\mathrm{X}$ ” and circle) are binned at the liquid/liquid interface of the AMPS (A and B). When the cuvette is near the top of the MagLev device white polystyrene beads (black "X" and circle) are captured at the liquid/liquid interface (C and D). Plots of the density versus position demonstrate illustrate the shift in the position of the gradient along the density axis (A and C). Dashed lines depict the liquid/air, liquid/liquid, and liquid/solid interfaces of an AMPS at the lower (A) and upper (C) positions. Images from experiments demonstrate the shift in the step in density by capturing Nylon beads in the lower position (B) and polystyrene beads in the upper position (D).

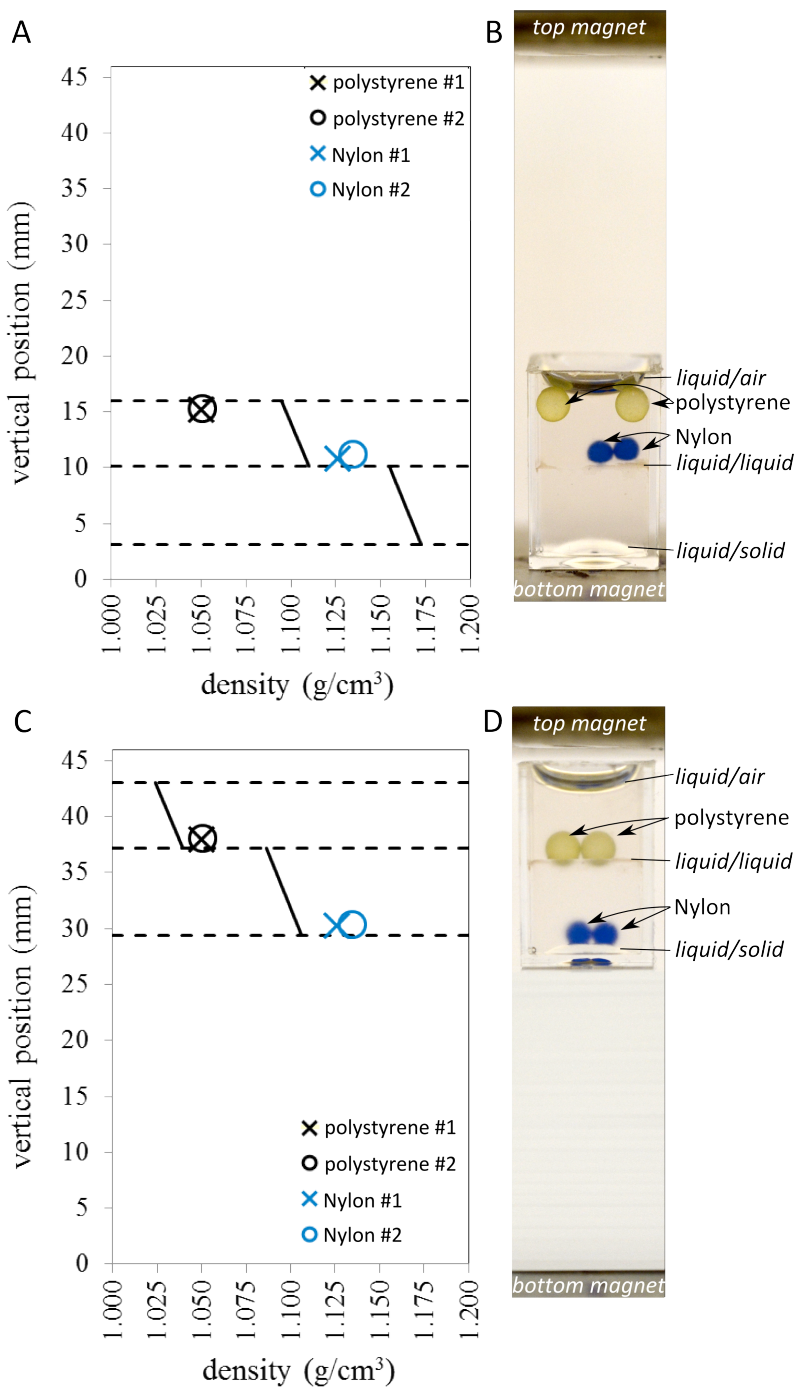


polystyrene and Nylon. By combining MagLev with AMPS, we enable the dynamic adjustment of the step in density that allows binning to sort plastics.

The AMPS we selected for this demonstration, like the other PEG-dextran system we describe, has a similar magnetic susceptibility in each phase; hence, as the position of the step in density changes, the size of the step remains constant. Using a system like the PEG$\mathrm{MnSO}_{4}$ AMPS described in this work with dissimilar magnetic susceptibilities in each phase would allow the height of the interface in the MagLev device to alter both the position and the size of the step in density.

\section{Conclusions}

Density is a characteristic of materials that is under-exploited for analysis and separation. Current gradients in density that allow separations based on density, however, typically cannot access multiple ranges without adjusting solutions, and multiple procedures may be required to isolate or analyze a desired object. Combining step gradients and linear gradients in density in fluids provides a new method to separate sub-populations from mixtures. We demonstrated this approach using aqueous multiphase systems (AMPS) and magnetic levitation (MagLev). In these systems, the density and magnetic susceptibility of a phase can be controlled independently to create tunable, stable media for separations based on density. AMPS prepared with more than two phases would enable separations over additional regions of density. Adding phases, however, also limits the total volume of each phase that is available to levitate objects.

The range of objects that can be separated with this method has some limitations. Because all phases in an AMPS contain water and a denser polymer, densities below the density of water $\left(0.998 \mathrm{~g} / \mathrm{cm}^{3}\right)$ are not accessible without adding a miscible organic cosolvent (e.g., ethanol or tetrahydrofuran). Densities as high as $1.300 \mathrm{~g} / \mathrm{cm}^{3}$ are accessible with the PEG-MnSO ${ }^{4}$ system described in this work. This range of densities is sufficient to 
cover at least part of the range of interest for many of the materials separated by density described in Table 1. The configuration of the MagLev device that we use in this work is also limited to separating objects with a characteristic size larger than $2 \mu \mathrm{m} .{ }^{10}$ Introducing centrifugation, increasing the strength of the magnets, or changing the geometry could all provide means to separate smaller objects with this approach. ${ }^{10}$ Due to the placement of the magnet above the container, extraction of samples separated in paramagnetic AMPS in MagLev is not as simple as extraction of objects separated in AMPS alone-where a simple pipette can be used to extract samples from an interface. We have, however, previously demonstrated methods to remove objects separated by MagLev including the use of syringes and tubing with a modified container ${ }^{49}$ and the use of a continuous flow system. ${ }^{50}$ These methods could be applied to a system that combines MagLev and AMPS, but a low flow rate may be required to maintain a stable interface between phases in a continuous flow method.

Combining MagLev and AMPS also provides new ways to use these techniques. The combination could extend the potential uses of AMPS by, in effect, making steps in density whose size can be adjusted with the introduction of a magnetic field gradient. The combination also allows new uses for MagLev. One limitation of MagLev in a homogenous solution with a linear field gradient is that solutions that achieve a large range ( $\Delta \rho$ over the distance between the magnets) will achieve a low sensitivity $(\Delta \rho / \Delta h$ where $h$ is the vertical distance). Although this same limitation exists for each phase of a paramagnetic AMPS, users can choose phases with different balances of range and sensitivity. This advantage of

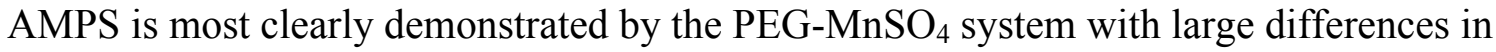
magnetic susceptibility between phases. Combining steps in density with linear gradients in density allows multiple ranges of density to be explored with different resolutions.

\section{Acknowledgements}


The authors thank Prof. Elena Rybak-Akimova (Tufts University) for helpful discussions about the Evans method. This work was supported by the Blavatnik Biomedical Accelerator Fund at Harvard University and by Tufts University. M.G. acknowledges support from the Marie Curie project SAM-TunEGaIn:IOF-2012-328412 (M.G.). A.A.K. was supported by an NSF Graduate Research Fellowship. J.A.W. was supported by a U.S. Department of Education GAANN fellowship and Tufts University. C.R.M was supported by Tufts University and the Bill and Melinda Gates Foundation (OPP1016360).

\section{Supporting Information Available}

The Supporting Information contains a detailed Materials and Methods section. Characterizations of the aqueous multiphase systems and the magnetic susceptibility are provided. A description of the methods used to measure the height of levitating beads and to estimate uncertainty in calculated densities is also provided. This information is available free of charge via the Internet at http://pub.acs.org/.

\section{References}

(1) Barth, H. G.; Boyes, B. E.; Jackson, C. Anal. Chem. 1996, 68, 445-466.

(2) Radisic, M.; Iyer, R. K.; Murthy, S. K. Int. J. Nanomedicine 2006, 1, 3-14.

(3) Julius, M. H.; Masuda, T.; Herzenberg, L. A. Proc. Natl. Acad. Sci. U. S. A. 1972, 69, 1934-1938.

(4) Miltenyi, S.; Müller, W.; Weichel, W.; Radbruch, A. Cytometry 1990, 238, 231-238.

(5) Pappas, D.; Wang, K. Anal. Chim. Acta 2007, 601, 26-35.

(6) Hage, D. Clin. Chem. 1999, 615, 593-615.

(7) Al-Salem, S. M.; Lettieri, P.; Baeyens, J. Waste Manag. 2009, 29, 2625-2643.

(8) Lockett, M. R.; Mirica, K. A.; Mace, C. R.; Blackledge, R. D.; Whitesides, G. M. J. Forensic Sci. 2013, 58, 40-45. 
(9) Pertoft, H. J. Biochem. Biophys. Methods 2000, 44, 1-30.

(10) Mirica, K. A.; Shevkoplyas, S. S.; Phillips, S. T.; Gupta, M.; Whitesides, G. M. J. Am. Chem. Soc. 2009, 131, 10049-10058.

(11) Arrowood, M.; Sterling, C. J. Parasitol. 1987, 73, 314-319.

(12) Mace, C. R.; Akbulut, O.; Kumar, A. A.; Shapiro, N. D.; Derda, R.; Patton, M. R.; Whitesides, G. M. J. Am. Chem. Soc. 2012, 134, 9094-9097.

(13) Pretlow, T. P. T. G. Methods 1991, 2, 183-191.

(14) Arnold, M. S.; Green, A. A.; Hulvat, J. F.; Stupp, S. I.; Hersam, M. C. Nat. Nanotechnol. 2006, 1, 60-65.

(15) Pollard, E.; Grady, L. Biophys. J. 1967, 205-213.

(16) Martínez-Salas, E.; Martín, J. A.; Vicente, M. J. Bacteriol. 1981, 147, 97-100.

(17) Tat, M. E.; Van Gerpen, J. H. J. Am. Oil Chem. Soc. 2000, 77, 115-119.

(18) Grigor'ev, B. A.; Ovchinnikov, N. A. High Temp. 2010, 48, 47-51.

(19) Park, J.-M.; Lee, J.-Y.; Lee, J.-G.; Jeong, H.; Oh, J.-M.; Kim, Y. J.; Park, D.; Kim, M. S.; Lee, H. J.; Jin Ho Oh; Lee, S. S.; Lee, W.-Y.; Huh, N. Anal. Chem. 2012, 84, 74007407.

(20) Kumar, A. A.; Patton, M. R.; Hennek, J. W. J. W.; Lee, S. Y. R. S. Y. R.; D’AlesioSpina, G.; Yang, X.; Kanter, J.; Shevkoplyas, S. S.; Brugnara, C.; Whitesides, G. M.; Wang, X. Proc. Natl. Acad. Sci. U. S. A. 2014, 111, 14864-14869.

(21) Kumar, A. A.; Lim, C.; Moreno, Y.; Mace, C. R.; Syed, A.; Tyne, D. Van; Wirth, D. F.; Duraisingh, M. T.; Whitesides, G. M. Am. J. Hematol. 2015, 90, 31-36.

(22) Feng, Y.; Miyata, Y.; Matsuishi, K.; Kataura, H. J. Phys. Chem. C 2011, 115, 17521756.

(23) Ghosh, S.; Bachilo, S. M.; Weisman, R. B. Nat. Nanotechnol. 2010, 5, 443-450.

(24) Mirica, K. A.; Phillips, S. T.; Mace, C. R.; Whitesides, G. M. J. Agric. Food Chem. 2010, 58, 6565-6569.

(25) Sparks, D.; Smith, R.; Straayer, M.; Cripe, J.; Schneider, R.; Chimbayo, a; Anasari, S.; Najafi, N. Lab Chip 2003, 3, 19-21.

(26) Grieve, M. J. Forensic Sci. Soc. 1987, 27, 405-412.

(27) Sakkas, D.; Manicardi, G. C.; Tomlinson, M.; Mandrioli, M.; Bizzaro, D.; Bianchi, P. G.; Bianchi, U. Hum. Reprod. 2000, 15, 1112-1116. 
(28) Chen, M. J.; Bongso, A. Hum. Reprod. 1999, 14, 759-764.

(29) Mason, C. B.; Bricker, T. M.; Moroney, J. V. Nat. Protoc. 2006, 1, 2227-2230.

(30) Bozidis, P.; Williamson, C. D.; Colberg-Poley, A. M. Curr. Protoc. Cell Biol. 2007, Chapter 3, Unit 3.27.

(31) Hennek, J. W.; Nemiroski, A.; Subramaniam, A. B.; Bwambok, D. K.; Yang, D.; Harburg, D. V; Tricard, S.; Ellerbee, A. K.; Whitesides, G. M. Adv. Mater. 2015, DOI: 10.1002/adma.201405207.

(32) Mirica, K. A.; Phillips, S. T.; Shevkoplyas, S. S.; Whitesides, G. M. J. Am. Chem. Soc. 2008, 130, 17678-17680.

(33) Bell, L. S.; Cox, G.; Sealy, J. Am. J. Phys. Anthropol. 2001, 116, 66-79.

(34) Richard, G. M.; Mario, M.; Javier, T.; Susana, T. Resour. Conserv. Recycl. 2011, 55, $472-482$.

(35) Subramaniam, A. B.; Yang, D.; Yu, H.-D.; Nemiroski, A.; Tricard, S.; Ellerbee, A. K.; Soh, S.; Whitesides, G. M. Proc. Natl. Acad. Sci. U. S. A. 2014, 111, 12980-12985.

(36) Albertsson, P.-Å. Biochim. Biophys. Acta 1958, 27, 378-395.

(37) Beijerinck, M. W. Zentralbl. Bakteriol. 1896, 2, 698.

(38) Akbulut, O.; Mace, C. R.; Martinez, R. V; Kumar, A. A.; Nie, Z.; Patton, M. R.; Whitesides, G. M. Nano Lett. 2012, 12, 4060-4064.

(39) Albertsson, P.-Å. Partition of Cell Particles and Macromolecules; Wiley Interscience: News York, 1986.

(40) Partitioning in Aqueous Two-Phase Systems: Theory, Methods, Uses, and Applications to Biotechnology; Walter, H., Brooks, D. E., Fisher, D., Eds.; New York, 1986.

(41) Zaslavsky, B. Y.; Mahmudov, A. U.; Bagirov, T. O.; Borovskaya, A. A.; Gasanova, G. Z.; Gulaeva, N. D.; Levin, V. Y.; Mestechkina, N. M.; Miheeva, L. M.; Rodnikova, M. N. Colloid Polym. Sci. 1987, 265, 548-552.

(42) Soohoo, J. R.; Walker, G. M. Biomed. Microdevices 2009, 11, 323-329.

(43) Kenkare, P. U.; Hall, C. K. AIChE J. 1996, 42, 3508-3522.

(44) Hernandez-Justiz, O.; Fernandez-Lafuente, R.; Terreni, M.; Guisan, J. M. Biotechnol. Bioeng. 1998, 59, 73-79.

(45) Hatti-Kaul, R. Mol. Biotechnol. 2001, 19, 269-277.

(46) Kahn, O. Molecular Magnetism, 1st ed.; Wiley-VCH: New York, 1993. 
(47) Evans, D. J. Chem. Soc. 1959, 2003-2005.

(48) Schubert, E. J. Chem. Educ. 1992, 69, 62.

(49) Atkinson, M. B. J.; Bwambok, D. K.; Chen, J.; Chopade, P. D.; Thuo, M. M.; Mace, C. R.; Mirica, K. A.; Kumar, A. A.; Myerson, A. S.; Whitesides, G. M. Angew. Chemie Int. Ed. 2013, 52, 10208-10211.

(50) Winkleman, A.; Perez-Castillejos, R.; Gudiksen, K. L.; Phillips, S. T.; Prentiss, M.; Whitesides, G. M. Anal. Chem. 2007, 79, 6542-6550. 
For Table of Contents (TOC) Only

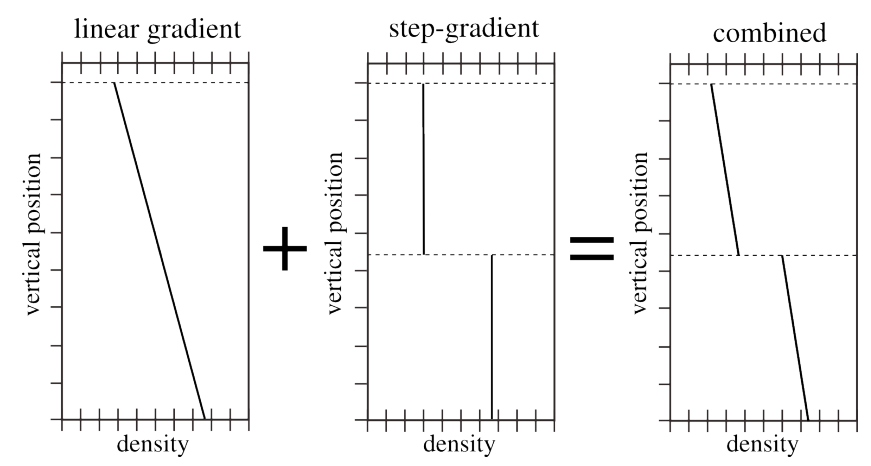

